\title{
THE UNCERTAINTY RELATION IN DISCRETE QUANTUM MECHANICS
}

\author{
Ting-Wai CHIU \\ Physics Department, National Taiwan University, Taipei, Taiwan 107, Republic of China
}

and

Friday LIN

Physics Department, National Tsing - Hua University, Hsin Chu, Taiwan 300, Republic of China

Received 31 December 1984

\begin{abstract}
The uncertainty relation in discrete quantum mechanics is obtained up to the lowest order correction to the continuum one. The distinguishing feature of this new uncertainty relation is that the minimal uncertainty is not only a function of the fundamental constant $l$ of the discreteness of the theory, but also a function of the dynamics of the system, in contrast to the usual minimal uncertainty $(h / 2)$ which is the same for all dynamical systems in continuum quantum mechanics.
\end{abstract}

The possibility that time can be regarded as a discrete dynamical variable has been explored by Lee [1]. Applying this concept to the quantum theory of physics, discrete quantum mechanics has been developed [2].

In this new mechanics there is no hamiltonian or lagrangian but only action. Thus the natural starting point is to use the path integration as the quantization procedure.

The Green function of a single particle in discrete quantum mechanics is expressed by the following sum over all discrete dynamical paths leading from the initial measurement $\left(\boldsymbol{x}_{0}, 0\right)$ to the final measurement $\left(x_{\mathrm{f}}, T\right)$ :

$$
\begin{gathered}
\left\langle\boldsymbol{x}_{\mathrm{f}}|G(T, l)| x_{0}\right\rangle=\sum_{N=0}^{\infty} \frac{1}{N !}\left(\frac{\mathrm{i}}{l}\right)^{N} \\
\times \int \prod_{n=1}^{N} \mathrm{~d} t_{n} \mathrm{~d}^{3} x_{n} \mathrm{e}^{\mathrm{i} A N},
\end{gathered}
$$

where $l$ is the fundamental constant of the theory, $J$ is the jacobian and $A_{N}$ is the discrete action for $N$ intermediate measurements given by

$$
\begin{aligned}
A_{N} & =\sum_{n=1}^{N+1}\left\{\frac{1}{2}\left(x_{n}-x_{n-1}\right)^{2} /\left(t_{n}-t_{n-1}\right)\right. \\
& \left.-\frac{1}{2}\left(t_{n}-t_{n-1}\right)\left[V\left(x_{n}\right)+V\left(x_{n-1}\right)\right]\right\} .
\end{aligned}
$$

The classical trajectory of the particle is determined by the following $4 N$ equations:

$\partial A_{N} / \partial x_{n}=0, \quad \partial A_{N} / \partial t_{n}=0$,

where $n=1,2, \ldots, N$. The most remarkable result of discrete classical mechanics is [1]: the time spacings between two successive measurements are of varying lengths for the oscillator potential, which is a general property for more complicated potentials. That means Newton's concept of time, which is absolute, flowing at a constant rate, and unaffected by the material objects, has undergone a fundamental change in discrete classical mechanics.

In this letter, we attempt to find the basic difference between the continuum and the discrete version of quantum mechanics. As we know, the uncertainty relation

$\Delta p \Delta x \geqslant \hbar / 2$

is one of the fundamental principles of the continuum quantum mechanics. However, the corresponding uncertainty relation in discrete quantum mechanics has not been rigorously derived yet. Although in ref. [1] the symmetrical discrete version of uncertainty relations has been written as

$\Delta p_{n} \Delta x_{n} \geqslant \hbar / 2$,

and 
$\Delta E_{n} \Delta t_{n} \geqslant \hbar / 2$,

where $p_{n}$ and $E_{n}$ are the eigenvalues of $-\mathrm{i} \hbar \partial / \partial x_{n}$ and $\mathrm{i} \partial \partial / \partial t_{n}$ respectively. Nevertheless, the physical measurements always refer to the coarse grain averages of $p_{n}$ and $E_{n}$ respectively. Therefore, the physical meaning of the uncertainty relations (5) and (6) is not clear to us. The main purpose of our present paper is to obtain the corresponding uncertainty relation in discrete quantum mechanics, which has the bearings on the physical measurements and at the same time agrees with the usual uncertainty relation (4) in the continuum limit $(l \rightarrow 0)$.

At first sight, it seems not possible to do so because there is no hamiltonian or lagrangian but only action (2) in the path integral (1) which is only a function of the dynamical variables $x_{n}$ and $t_{n}$. Fortunately, an effective hamiltonian $H$ of a particle (with unit mass and the simplest choice of $I$ ) has been derived in ref. [2]:

$H=K+V+\frac{1}{2} l^{2}\left[K+\frac{1}{2} V,[K, V]\right]+O\left(l^{3}\right)$,

$$
K=-\frac{1}{2} \nabla^{2} \text {. }
$$

Now, in order to get the corresponding uncertainty relation, we define the effective momentum operator $\boldsymbol{P}$ and the effective position operator $\boldsymbol{X}$ by the following relation:

$H=H(P, X)=\frac{1}{2} P^{2}+V(X)$.

Since the eigenvalue of the effective hamiltonian $H$ is the coarse grain average of $E_{n}$, then it can be shown that the eigenvalues of the effective momentum $P$ and the effective coordinate $X$ are the coarse grain averages of $p_{n}$ and $\boldsymbol{x}_{n}$ respectively.

For simplicity, we only consider the one-dimensional case. The extension to three dimensions is trivial. Combining (7) and ( 8 ) gives

$$
\begin{aligned}
& \frac{1}{2} P^{2}+V(X)=K+V+\frac{1}{2} l^{2}\left[K+\frac{1}{2} V,[K, V]\right] \\
& \quad+O\left(l^{3}\right) .
\end{aligned}
$$

Because the term of the first order in $l$ is absent on the right hand side of (9), it is simple to find that ( $p \equiv$ $-\mathrm{i} \partial / \partial x)$

$P=p-\frac{1}{4} l^{2}\left[p V^{\prime \prime}(x)+V^{\prime \prime}(x) p\right]+\mathrm{O}\left(l^{3}\right)$

and

$X=x+\frac{1}{4} l^{2} V^{\prime}(x)+\mathrm{O}\left(l^{3}\right)$, where $V^{\prime}$ and $V^{\prime \prime}$ represent first and second derivatives of the potential. Therefore, the commutation relation of $P$ and $X$ is

$[P, X]=-\mathrm{i}\left[1-\frac{1}{4} l^{2} V^{\prime \prime}(x)\right]+\mathrm{O}\left(l^{3}\right)$,

and the lowest order correction to the uncertainty relation (4) follows immediately,

$\Delta P \Delta X \geqslant \frac{1}{2}\left[1-\frac{1}{4} l^{2}\left\langle V^{\prime \prime}(x)\right\rangle\right]+\mathrm{O}\left(l^{3}\right)$,

where $\left\langle V^{\prime \prime}(x)\right\rangle$ is the expectation value of $V^{\prime \prime}(x)$. This is the main result of our paper. It is instructive to consider the oscillator potential $V(x)=\frac{1}{2} \omega^{2} x^{2}$, then (13) yields the uncertainty relation

$$
\Delta P \Delta X \geqslant \frac{1}{2}\left(1-\frac{1}{4} l^{2} \omega^{2}\right)+O\left(l^{3}\right) \text {, }
$$

which implies that the zero point energy of the oscillator is

$\frac{1}{2} \omega\left(1-\frac{1}{4} l^{2} \omega^{2}\right)+O\left(l^{3}\right)$,

consistent with the result in ref. [2].

It is evident that (13) agrees with the usual uncertainty relation in the continuum limit $(l \rightarrow 0)$. We also note that eq. (12) reduces to the usual commutator of the momentum operator and the position operator in the continuum limit. It is interesting to remark that the minimal uncertainty in discrete quantum mechanics depends on the dynamics of the system. This situation is quite different from the case of continuum quantum mechanics where the uncertainty relation is only a kinematic constraint for all systems. This kinematic constraint is closely related to the waveparticle duality nature of matter. Now, in discrete quantum mechanics the time has been regarded as a discrete dynamical variable and thus as part of matter. This must change the kinematic constraint to a dynamical one.

This research is supported in part by National Science Council R.O.C. We would like to thank Professor K.Y. Lin at National Tsing-Hua University for reading the manuscript.

\section{References}

[1] T.D. Lee, Phys. Lett. 122B (1983) 217; Discrete mechanics, in: Proc. Intern. School of Subnuclear physics (Erice, 1983), to be published.

[2] R. Friedberg and T.D. Lee, Nucl. Phys. B225 (1983) 1. 